\title{
The relationship between white matter hyperintensities and cognitive reference abilities across the life span
}

\author{
Ana R. Moura ${ }^{\mathrm{a}, \mathrm{b}}$, Seonjoo Lee ${ }^{\mathrm{c}, \mathrm{d}, \mathrm{e}}$, Christian Habeck ${ }^{\mathrm{a}}$, Qolamreza Razlighi ${ }^{\mathrm{a}}$, \\ Yaakov Stern ${ }^{\mathrm{a}, *}$ \\ ${ }^{a}$ Cognitive Neuroscience Division, The Taub Institute for Research on Aging and Alzheimer's Disease, Columbia University, New York, NY, USA \\ ${ }^{\mathrm{b}}$ Departamento de Psiquiatria e Saúde Mental, Centro Hospitalar Lisboa Ocidental, Lisboa, Portugal \\ ${ }^{\mathrm{c}}$ Mental Health Data Science, New York State Psychiatric Institute, New York, NY, USA \\ ${ }^{\mathrm{d}}$ Department of Biostatistics, Columbia University, New York, NY, USA \\ ${ }^{\mathrm{e}}$ Department of Biostatistics and Psychiatry, Columbia University, New York, NY, USA
}

\section{A R T I C L E I N F O}

\section{Article history:}

Received 28 March 2019

Received in revised form 12 August 2019

Accepted 24 August 2019

Available online 29 August 2019

Keywords:

White matter hyperintensities

MRI

Cognitive aging

Adult lifespan

\begin{abstract}
A B S T R A C T
We examined the relationship between white matter hyperintensities (WMH) burden and performance on 4 reference abilities: episodic memory, perceptual speed, fluid reasoning, and vocabulary. Crosssectional data of 486 healthy adults from 20 to 80 years old enrolled in an ongoing longitudinal study were analyzed. A piecewise regression across age identified an inflection point at 43 years old, where WMH total volume began to increase with age. Subsequent analyses focused on participants above that age $(\mathrm{N}=351)$. WMH total volume had significant inverse correlations with perceptual speed and memory. Regional measures of WMH showed inverse correlations with all reference abilities. We performed principal component analysis of the regional WMH data to create a model of principal components regression. Parietal WMH regional volume burden mediated the relationship between age and perceptual speed in simple and multiple mediation models. The principal components regression pattern associated with perceptual speed also mediated the relationship between age and perceptual speed performance. These results across the extended adult life span help clarify the influence of WMH on cognitive aging.
\end{abstract}

(c) 2019 Elsevier Inc. All rights reserved.

\section{Introduction}

White matter hyperintensities (WMH), which are distributed patches of augmented lucency (bright signal) on T2-weighted magnetic resonance imaging (MRI) are a common finding in brain scans of older people (Longstreth et al., 1996; van Dijk et al., 2002). Clinically, there is evidence that WMH are associated with cognitive decline and may have a role in the etiology of dementia (Brickman et al., 2009; de Groot et al., 2001; De Groot et al., 2002; De Leeuw et al., 2001; DeCarli et al., 2005; Frisoni et al., 2007; Iadecola, 2013; Yoshita et al., 2006). However, in the clinical setting, WMH are often seen as benign comorbidity of aging due to their very high prevalence in older adults (Lindemer et al., 2017a,b).

To better understand the impact of WMH in the aging brain, it is important to explore their association with cognitive domains that are affected by aging and dementia. For example, Breteler et al. reported that periventricular WMH, brain infarcts, and generalized

\footnotetext{
* Corresponding author at: 630 W 168th St, P\&S Box 16, New York, NY 10032. Tel.: 212-342-1350; fax: 212-342-1838.

E-mail address: ys11@columbia.edu (Y. Stern).
}

brain atrophy on MRI are associated with the rate of decline in processing speed and executive function (Prins et al., 2005). In addition, it is important to consider the localization of WMH. For example, it has been reported that parietal WMH are associated with a higher risk of developing Alzheimer's disease (Brickman et al., 2015, 2012), whereas frontal WMH are associated with higher risk of mortality (Wiegman et al., 2013). In samples of community-dwelling middleage adults, specific brain loci (clusters) of WMH burden were inversely correlated with episodic memory (Smith et al., 2011) and executive function (Smith et al., 2011).

In this article, we first used a piecewise regression across age to establish an inflection point where WMH burden increased systematically and limited our analysis to above that age. We focused on the relationship between WMH burden and performance on 4 cognitive domains, or reference abilities (RAs), that capture most of the age-related variance in cognitive performance (Salthouse, 2005). Finally, we used mediation models to explore whether WMH mediated the relationship between the effects of age and each RA. In healthy adults aged $20-80$ years, we explored the patterns of regional WMH burden that best predicted cognitive performance. 


\section{Materials and methods}

\subsection{Participants}

Data for the present study was collected in 2 ongoing projects-the Reference Ability Neural Networks and the Cognitive Reserve (CR) studies. The Reference Ability Neural Networks study was designed to identify neural networks associated with cognitive performance throughout the life span of a healthy sample in the 4 previously mentioned RAs: episodic memory, perceptual speed, fluid reasoning, and vocabulary (Salthouse, 2009). The CR study aims to elucidate the neural underpinnings of cognitive reserve and clarify the concept of brain reserve from a neuroimaging perspective (Stern, 2012). Detailed information about the 2 studies is provided in previous publications (Habeck et al., 2016; Stern, 2012; Stern et al., 2014). Only the WMH neuroimaging data and neuropsychological test scores from these studies were used in the present study.

This study uses data from cognitively healthy adults aged between 18 and 80 years. Recruitment was carried out using a random market mailing procedure within 10 miles of the Columbia University Medical Center. This recruitment approach intends to obviate cohort effects that might be present by using convenience samples. Participants were telephone-screened as the first part of the recruitment process. The screening phase checked the basic inclusion criteria, which included being right-handed, native English speaker, have at least fourth grade reading level. If the participants passed the first level of screening, they underwent an inperson assessment. Older subjects were screened to insure they did not meet criteria for dementia or mild cognitive impairment and had a score greater than 130 on the Mattis Dementia Rating Scale (Mattis, 1988). Other exclusion criteria were as follows: any psychiatric history, namely psychosis, recent (past 5 years) major depressive, bipolar, or anxiety disorders, electroconvulsive therapy treatment, alcohol or drug abuse within the last 12 months; cardiovascular diseases such as myocardial infarction, congestive heart failure, or any other cardiovascular disease; neurological disorders such as stroke, tumor, central nervous system infection, epilepsy, multiple sclerosis, degenerative diseases, head injury (loss of consciousness $>5$ minutes), mental retardation, seizure, Parkinson's disease, Huntington's disease, normal pressure hydrocephalus, essential/familial tremor, untreated neurosyphilis, Down syndrome; uncontrolled hypertension, uncontrolled diabetes mellitus or insulin-dependent diabetes, uncontrolled thyroid or other endocrine disease; uncorrectable vision, color blindness, uncorrectable hearing and implant; pregnancy, lactating; any medication targeting central nervous system; oncological disease within the last 5 years; renal insufficiency and/or active hepatic disease. No participant was excluded based on his or her WMH volume.

The Institutional Review Board of the College of Physicians and Surgeons of Columbia University approved the study. The consent form was obtained before study participation and after the nature and risks of the study were explained. All subjects received compensation for their participation.

\subsection{Measures}

\subsubsection{Reference abilities}

Based on a previous principal axis factor analysis (Salthouse et al., 2015), a composite score for each of the RAs was obtained using measures from a battery of well-established neuropsychological instruments. Z-scores were calculated for each cognitive task. For each RA (representing a cognitive domain), a summary score was calculated by averaging the 3 z-scores. Higher scores indicate better performance. The 4 domains include the following tests:

Fluid reasoning. This composite score was based on 3 tests, the Wechsler Adult Intelligence Scale-III (WAIS-III) Block Design test (Wechsler, 1997), in which participants are asked to reproduce a series of increasingly complex geometrical shapes using 4 or 9 identical blocks with red, white, or split red and white sides; WAISIII Letter-number Sequencing (Wechsler, 1997), in which participants are asked to recall progressively longer lists of intermixed letters and numbers first in alphabetical and then numerical order; and WAIS-III Progressive Matrices, in which participants are asked to select which pattern in a set of 8 possible patterns best completes a missing cell in a matrix.

Vocabulary. The composite score was based on the vocabulary subtest from the WAIS-R (Wechsler, 1997), which asks participants to provide definitions for series of increasingly more complex words, as well as the Wechsler Test of Adult Reading (Wechsler, 2001) and the American National Adult Reading Test (Grober and Sliwinski, 1991) that both involve pronouncing irregularly spelled English words.

Episodic memory. The composite score was based on 3 subscores obtained through Selective Reminding Test (SRT). In this task, participants initially read a list of 12 words and were asked to recall as many as they could. For the following 5 trials, they were reminded of the words that they did not report and were asked to again recall all the words in the list. Words are considered to enter long-term storage from the point when they are recalled twice in a row without reminders. The subscores used are Long-Term Storage Subscore (SRT LTS), the sum of all words recalled at least twice in a row, Consistent Long-term Retrieval (SRT CLRT), the sum of all words that are consistently recalled, finally, Last Trial (SRT Last), which is the number of words recalled on the last trial of the test (Buschke and Fuld, 1974).

Perceptual speed. The composite score was based on the 3 different tests, the WAIS-III Digit-symbol subtest (Wechsler, 1997), where participants were instructed to draw the symbol corresponding to a specific number as quickly as possible, the score is the number of correctly produced symbols in 90 seconds; Trail Making Test (TMT) Part A, where participants were asked to connect circles numbered from one to 24 as rapidly as possible, the score is the time to connect all 24 circles. (Reitan, 1958); Stroop Color Naming test, where the participants were asked to identify the colored ink patches, the score is the number of color ink patches named in 45 seconds (Golden, 1978).

\subsection{Imaging procedures}

\subsubsection{MRI acquisition and image processing}

All images were acquired on the same 3.0 T Philips Achieva Magnet. A coronal T1-weighted MPRAGE scan was used to determine the participant's position. Parameters for the EPI acquisition included the following: $3 \mathrm{msec}$ for the T1-weighted repetition time, 6.5 msec echo time, $8^{\circ}$ deg flip angle, $256 \times 200$ voxels in-plane resolution, and $165-180$ slices in the axial direction with slicethickness/gap of $1 / 0 \mathrm{~mm}$.

In addition, an FLAIR scan was acquired with the following parameters: $11,000 \mathrm{msec}$ repetition time, $2800 \mathrm{msec}$ echo time, $256 \times$ 189 voxels in-plane resolution, $23.0 \times 17.96 \mathrm{~cm}$ field of view, and 30 slices with slice-thickness/gap of $4 / 0.5 \mathrm{~mm}$. This sequence was used to quantify the $\mathrm{WMH}$ volumes. A neuroradiologist reviewed each scan individually to exclude any relevant findings. In the case of a 
clinical positive finding, the subject's primary care physician was informed.

\subsubsection{Image processing}

Structural T1 scans were reconstructed using FreeSurfer v5.1 (http://surfer.nmr.mgh.harvard.edu/). The accuracy of FreeSurfer's subcortical segmentation and cortical parcellation has been reported to be comparable with manual labeling (Desikan et al., 2006; Fischl et al., 2002). Each participant's boundaries of white and gray matter, as well as gray matter and cerebral-spinal-fluid boundaries, were visually inspected slice by slice, and manual control points were added in case of any visible discrepancy. Boundary reconstruction was repeated until reaching optimized results for every participant. The subcortical structure borders were plotted by FreeView visualization tools and manually reviewed. In the case of discrepancy, the manual correction was performed. Finally, we computed mean values for white matter (WM) cortical volumes and intracranial volumes (ICV) for each participant.

\subsubsection{Automatic WMH (total volumes) segmentation, classification, and quantification}

WMH were segmented by the Lesion Segmentation Tool algorithm (LST) (Schmidt et al., 2011) as implemented in the LST toolbox version 2.0.15 (June 2017) for Statistical Parametric Mapping (www. statistical-modelling.de/lst.html). The algorithm first segments the T1 images into the 3 main tissue classes-cerebral brain fluid, gray matter, and WM. Then, this information is combined with the coregistered FLAIR intensities to calculate lesion belief maps. By thresholding these maps with a prechosen initial threshold $(\kappa=$ 0.5 ), an initial binary lesion map is obtained which is subsequently grown along voxels that appear hyperintense in the FLAIR image. The result is a lesion probability map. Every FLAIR sequence that had a total WMH volume above $1000 \mathrm{~mm}^{3}$ was manually inspected to ensure that there were no visible discrepancies.

\subsubsection{Extraction of regional WMH volumes}

To extract lobar WMH values, we first registered the T1 sequence of each participant in FreeSurfer and then coregistered the FLAIR sequence. The following lobar regions, frontal, parietal, temporal, cingulate, and occipital, were defined as the lobes in FreeSurfer. The volumes of WMH in each of the 5 lobes were automatically extracted. One participant was excluded due to technical problems in extracting the regional WMH map.

\section{Statistical analysis}

We performed the statistical analyses in R 3.14.2 (The R Foundation for Statistical Computing), using base functions and mediation package. MATLAB R2017a, MathWorks, Natick, was used for the principal component analysis (PCA) and principal component regression (PCR). Descriptive statistics were calculated, including means and standard deviations, minimum and maximum absolute values, frequency counts and percentage for demographic and neuropsychological variables, and median and interquartile range for the neuroimaging variables. We used two-tailed significance tests, and $p$-values less than 0.05 was regarded as statistically significant. To reduce skewness, WMH values were transformed using an inverse hyperbolic sine (IHS). This transformation is used to reduce the influence of extreme observations and behaves like the logarithmic transformation for large enough values, regardless of its parameter value 0 (as long as it does not equal 0) (Burbidge et al., 1988).

To account for zero inflated distribution, we considered a twopart model (Belotti et al., 2015) using logistic regression to model having white matter hyperintensities (WMH) burden and lognormal regression to model the distribution of $\mathrm{WMH}$ when it is greater than zero. Given the hypothesized nonlinear relationship between age and WMH (total and regional volumes), we used a piecewise regression model (also called "broken stick" model) to study the trend of this relationship. We employed this model with an inflection point as a parameter. This regression model is often used when it is assumed that after a certain lag the response increases or decreases linearly, starting from the baseline value. This assumption reflects a model in which the first part is a horizontal line (McGee and Carleton, 1970). In piecewise linear regression, the inflection point was selected using the Bayesian information criterion. The likelihood ratio test was used to test whether the inclusion of the inflection point significantly improved the model fit, compared with the model without the inflection point (Piepho and Ogutu, 2003). All the subsequent analysis considered only ages above the calculated inflection point.

To explore the relationship between WMH (regional and total volumes) and the 4 RAs, we performed 2 different sets of linear regression models. First, we separately fit a linear regression model for each of the 4 RAs (as the dependent variable) with age and total $\mathrm{WMH}$ as independent variables. Second, we fit linear regression models for each of the 4 RAs (as the dependent variable) with age and the 5 regional $\mathrm{WMH}$ as independent variables. Education (in years), gender, and ICV were entered as covariates in the previously explained models.

We then applied PCA and PCR to the WMH values in the 5 lobes. More details about this multivariate decomposition technique can be found in the studies by Lee et al., 2016 and Habeck et al., 2008. The consideration of these techniques in these data reduces the chances of collinearities. We derived a covariance pattern, consisting of a set of principal components (PCs) that best predicted performance in each RA. We excluded one individual that had a volume of $0 \mathrm{~mm}^{3}$ of $\mathrm{WMH}$ in all 5 lobes. The procedure was as follows:

Derive a PCA on the data of the $5 \mathrm{WMH}$ regional volumes (IHS transformed) across all subjects with age above the inflection point. Each lobe (representing the WMH burden in that region) in each PC may have either a positive or a negative loading. These loadings are fixed and the same for all subjects. The expression of each PC for each subject was quantified by a subject-scaling factor or the "individual PC expression score". Therefore, the subject-scaling factor expresses the degree of subjects' expression of the fixed PC. To find a covariance pattern that best discriminates cognitive performance in each of the RAs, each subject's expression of the specified PC derived from step 1 was entered into a linear regression model as the independent variable. Each RA individually was the dependent variable. This regression results in a linear combination of the PC that best predicts cognitive performance. This linear combination of the PC can itself be conceived as signifying a "pattern" of WMH distribution. We used Akaike's information criterion to decide how many PCs should be included in the regression to achieve optimal bias-variance trade-off. The set of PCs that yields the lowest value in Akaike's information criterion was selected as predictors in the regression model. We did not restrict the number of PCs, so we checked the following sets: PC1, PC1-2, PC1-3, PC1-4, and PC1-5. PC1 was always included because combinations excluding PC1 had very small variance contributions and failed to show robust-enough loadings in the bootstrap procedure. The set that yielded the lowest value of Akaike information criterion was selected for construction of the discriminant pattern. For the pattern construction, after the optimal subset is selected, we took the regression weights for all the chosen components and constructed a corresponding pattern, which assigned a loading to each regional volume. For the bootstrap estimation of loading robustness, we performed a bootstrap resampling test with 10,000 iterations, meaning that each time we resampled the pool of subjects with replacement and repeated our analysis for the specific PC-set picked in the point estimate 
computation. For each lobe, the $95 \%$ confidence interval $(\mathrm{CI})$ was listed. This procedure provided information on the stability of the contribution from each WMH regional volume toward the covariance pattern. If the $95 \% \mathrm{CI}$ did not include the zero point, the loading can be interpreted as stable. We did not account for the possible nonlinear association between variables in this analysis.

Finally, we tested a formal mediation model between age, WMH total volume, and each of the 4 RAs. We started the mediation analysis by testing whether the primary independent variable, in this case age, predicted the dependent measure of each of the 4 RAs. Next, we tested the direct effects of age on the mediator (WMH total volume) and the direct relationship between the mediator, WMH total volume, and the dependent variable (cognition, as measured by the RA). Then we tested the indirect mediating effect or the extent to which the relationship between age and each of the 4 RAs has WMH total volume as a mediator. We hypothesized that there is a mediating effect of each regional WMH in the relationship between age and each of the 4 RAs. After running the model for WMH total volume, we also reran a mediation model with each of the regional $\mathrm{WMH}$ volumes as the mediators. We tested a mediation model using the 5 regional WMH volumes as multiple mediators and controlling for education. Finally, we tested a mediation model using PC-derived patterns.

\section{Data availability}

The data that support the findings of this study are available from the corresponding author, on request.

\section{Results}

\subsection{Demographics}

The total sample (from 20 to 80 years old) included 486 healthy adults who had the T1 and FLAIR scans. 468 completed the neuropsychological evaluation and 464 completed the memory tasks. The excluded participants could not complete the neuropsychological evaluation. Table 1 contains the sociodemographic characteristics, neuropsychological scores, and the MRI data of the participants included in the analysis.

\subsection{The relationship between age and $W M H$ total volume-piecewise linear regression}

Fig. 1 shows the relationship between $\mathrm{WMH}$ (total volume) and age for each individual included in the present study $(n=486)$. The Spearman's rank-order correlation coefficient between age and WMH total volume was $0.69(p<0.0001)$. Broadly, volumes larger than $1000 \mathrm{~mm}^{3}$ appear after 40 years of age, becoming increasingly larger for subjects older than 50 years. In fact, the relationship between age and WMH total volume before the age of 43 years was closer to a horizontal line, and after 43 years of age, this trend changes.

We fitted a piecewise regression model to better address the nonlinear trend of the association between age and WMH total volume. This model estimated the existence of one inflection point at 43 years of age ( $95 \% \mathrm{CI}$ between 24.35 and 59.10 years old). After determining the inflection point where the relationship between WMH total volume and age becomes closer to linearity, we restricted all subsequent analyses to participants above age 43 years.

Fig. 2 shows the average probability of having WMH in 4 age groups. The probability increases with age, and it is higher in the 70-80 year olds.

\subsection{Correlation of age, cognition, and WMH (total and regional volumes)}

Fig. 3 shows the correlation matrix for the variables of interest using Spearman's rank-order correlation. The sample included

Table 1

Demographic, neuropsychological, and imaging data

\begin{tabular}{|c|c|c|c|c|}
\hline \multirow[t]{2}{*}{ Variables } & \multicolumn{2}{|l|}{ Total sample $(n=486)$} & \multicolumn{2}{|c|}{ Sample above 43 y old $(n=351)$} \\
\hline & $\overline{\text { Mean (SD) }}$ & Min-max & Mean (SD) & Min-max \\
\hline \multicolumn{5}{|l|}{ Demographic data } \\
\hline Age (y) & $53.69(16.78)$ & 20.00 to 80.00 & $62.82(8.77)$ & 43 to 80 \\
\hline Gender (\% of women) & 56.2 & - & 67.1 & - \\
\hline Education $(y)$ & $16.16(2.37)$ & 9.00 to 24.00 & $16.26(2.40)$ & 11 to 22 \\
\hline \multicolumn{5}{|l|}{ Neuropsychological data } \\
\hline NARTIQ (mean z-score) & $117.07(8.73)$ & 85.28 to 130.88 & $118.89(8.46)$ & 85.28 to 130.28 \\
\hline \multicolumn{5}{|l|}{ Reference abilities (z-score) $)^{\mathrm{a}}$} \\
\hline 1. Fluid reasoning & $0.02(0.82)$ & -1.93 to 2.17 & $-0.17(0.74)$ & -1.93 to 2.03 \\
\hline 2. Vocabulary & $0.03(0.92)$ & -2.86 to 1.28 & $-0.19(0.91)$ & -2.50 to 1.60 \\
\hline 3. Episodic memory & $0.02(0.93)$ & -2.50 to 1.64 & $0.16(0.88)$ & -2.71 to 1.28 \\
\hline \multirow[t]{2}{*}{ 4. Perceptual speed } & $0.00(0.83)$ & -3.22 to 2.19 & $-0.22(0.74)$ & -3.22 to 1.66 \\
\hline & \multicolumn{4}{|c|}{ Median (interquartile range) } \\
\hline \multicolumn{5}{|l|}{ Imaging data } \\
\hline WMH volume $\left(\mathrm{mm}^{3}\right)$ & $114.50(690.00)$ & 0.00 to $51,917.0$ & 310.00 (1735.99) & 0.00 to $51,917.01$ \\
\hline WMH (IHS) & $5.43(4.44)$ & 0.00 to 11.55 & $6.43(3.66)$ & 0.00 to 11.55 \\
\hline Cortical WM total volume $\left(\mathrm{mm}^{3}\right)$ & $462198.31(73,841.00)$ & 304556 to 680077 & $458257.28(73,834.57)$ & 304555.6 to 615191.97 \\
\hline Intracranial volume $\left(\mathrm{mm}^{3}\right)$ & $1475467.00(197260.00)$ & 1042703 to 1900961 & $1473744.50(198505.2)$ & 1042703.0 to 1844558.00 \\
\hline \multicolumn{5}{|l|}{ WMH regional $(\mathrm{IHS})^{\mathrm{b}}$} \\
\hline 1. Frontal & $0.00(1.21)$ & 0.00 to 4.20 & $0.00(1.77)$ & 0.00 to 4.20 \\
\hline 2. Cingulate & $0.00(0.00)$ & 0.00 to 3.26 & $0.00(0.00)$ & 0.00 to 3.26 \\
\hline 3. Occipital & $1.21(2.23)$ & 0.00 to 3.46 & $1.61(2.40)$ & 0.00 to 3.46 \\
\hline 4. Temporal & $0.00(0.00)$ & 0.00 to 3.21 & $0.00(0.38)$ & 0.00 to 3.21 \\
\hline 5. Parietal & $0.00(1.30)$ & 0.00 to 3.76 & $0.00(1.72)$ & 0.00 to 3.76 \\
\hline
\end{tabular}

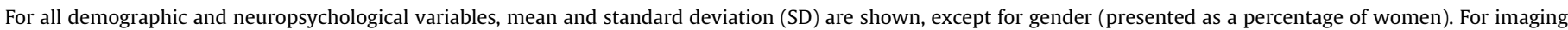
variables median and interquartile range are presented.

WMH total volume has an $\mathrm{n}=485$.

Key: NARTIQ, National Adult Reading Test Intelligence Quotient; WMH, white matter hyperintensities; IHS, inverse hyperbolic sine.

a All reference abilities have an $n=468$ except for episodic memory where it is 464 .

b Regional WMH volume burden has an $\mathrm{n}=350$. 

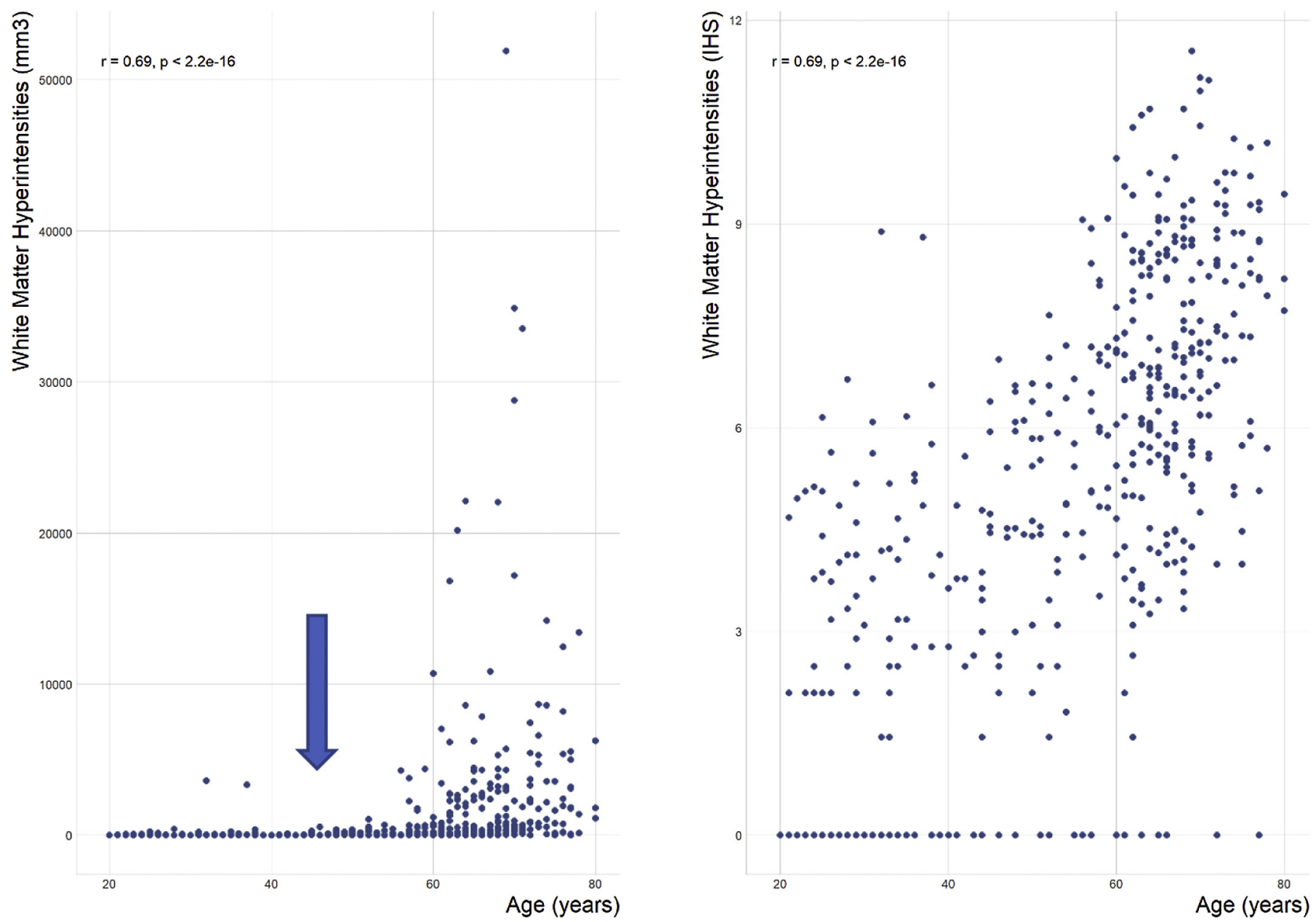

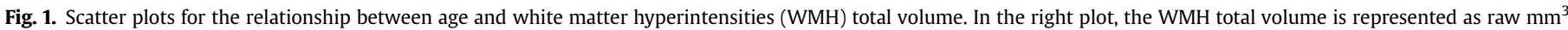

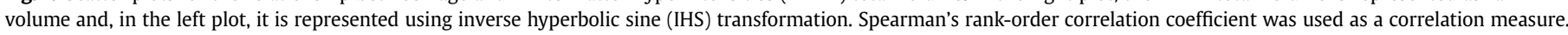

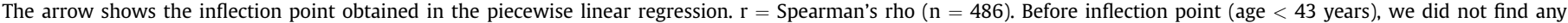
association $(\beta 1=0.16, p=0.76$ ), while after infection point, WMH was significantly associated with age $(\beta 2=4.52 ; p<0.0001)$.

participants aged 43 years or older $(\mathrm{N}=351)$. As expected, we found a moderate to a strong positive correlation between age and WMH total volume (Spearman's rho $=0.51, p<0.001$ ). WMH total volume had weak significantly inverse correlations with speed of

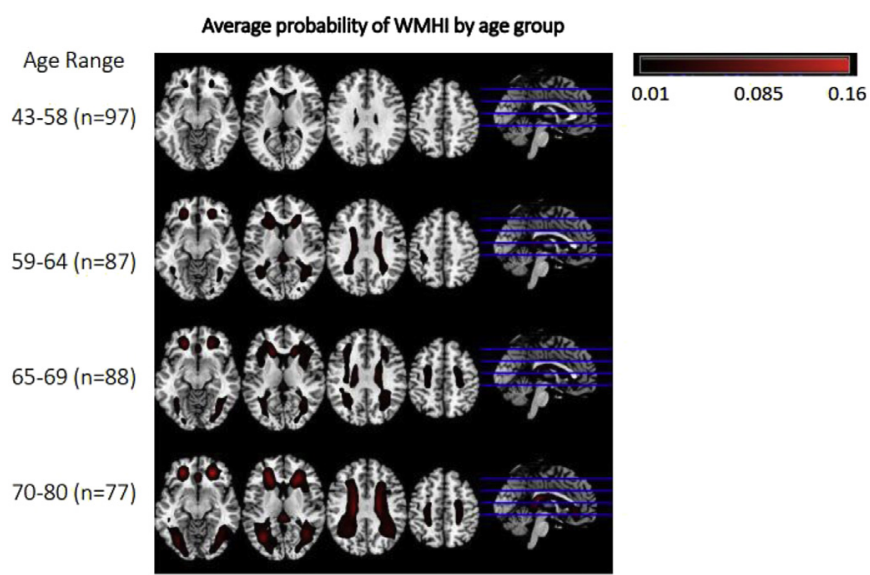

Fig. 2. Probability of having WMH in the different age groups above 43 years old. Abbreviations: WMH, white matter hyperintensities. processing (Spearman's rho $=-0.23, p<0.001$ ) and memory (Spearman's rho $=-0.16 p<0.001$ ). Regional measures of WMH showed weak inverse correlations with each of the 4 RAs, being the highest between parietal regional WMH and speed of processing RA (Spearman's rho $=-0.27, p<0.001$ ). In addition, the five-regional lobar WMH had significant positive correlations among themselves.

\subsection{The relationship between age, WMH total and regional volumes, and cognition}

Fig. 4 shows the correlation of WMH total volume with each of the 4 RAs. Simple linear regression models revealed significant associations between each of the 4 RAs and WMH (see Supplementary Table 1). When age was entered into the model, the associations lost their significance as depicted in Supplementary Table 2. Supplementary Table 3 contains the parameter estimates for the first 4 regression models with age and education as covariates. We reran these models controlling also for gender and ICV and similar results were obtained (data not shown).

Scatter plots showing the relationship between age and each of the $5 \mathrm{WMH}$ regional volumes are shown in Fig. 5. All the correlation coefficients are positive ( $p$-values $<0.001)$. In general, WMH volumes larger than 1 (IHS) appear after the fourth decade of life and became increasingly large after that age. The Spearman's rank- 


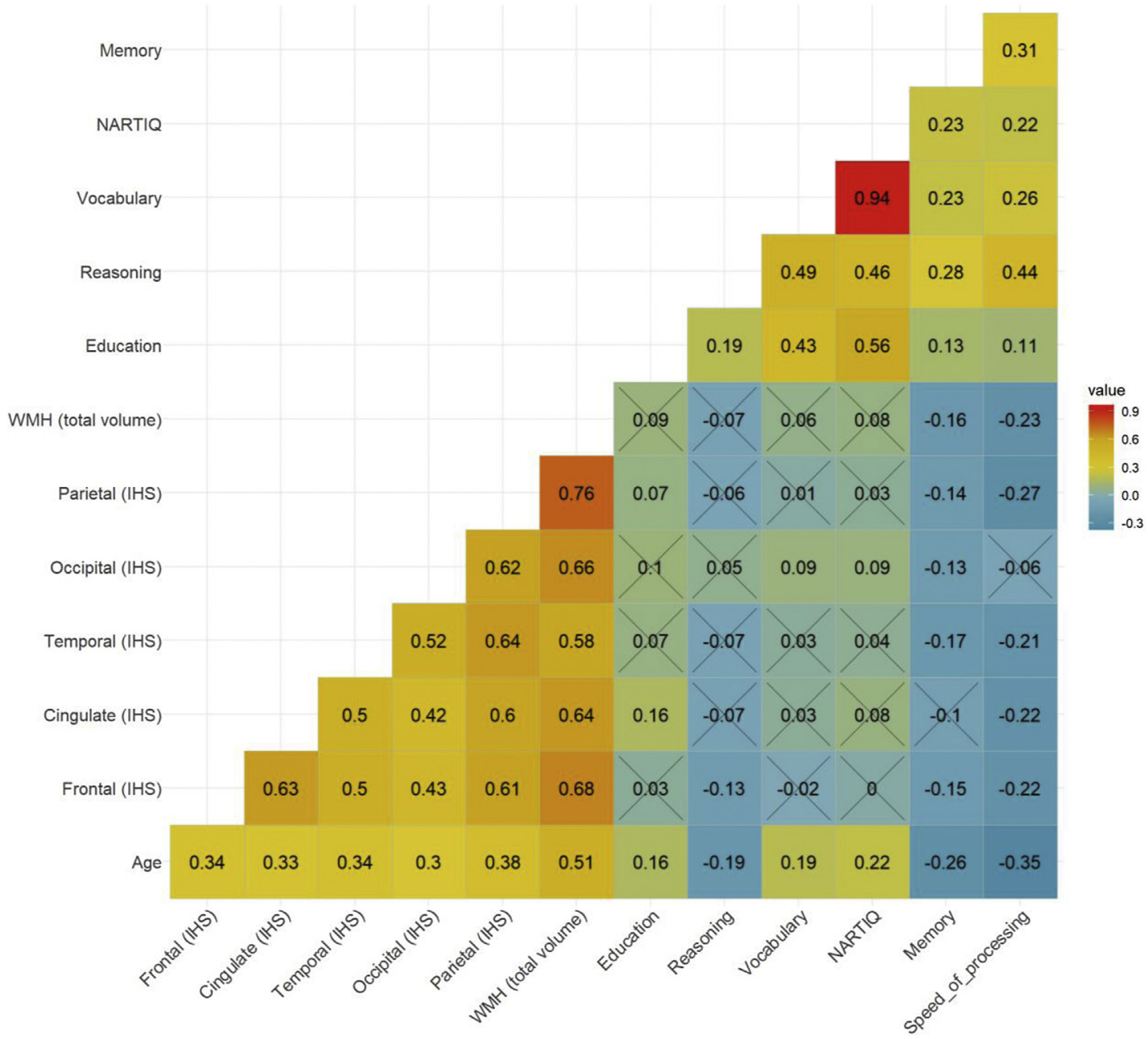

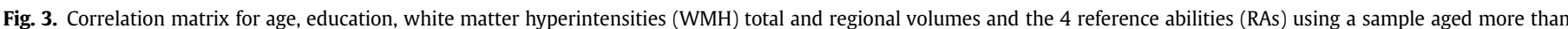

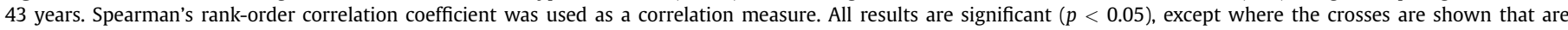

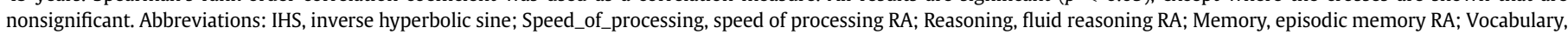

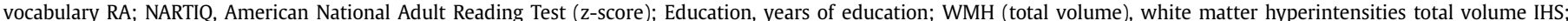

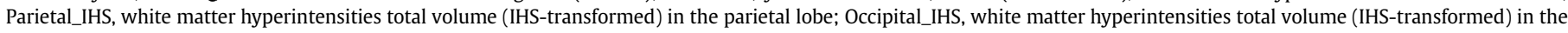

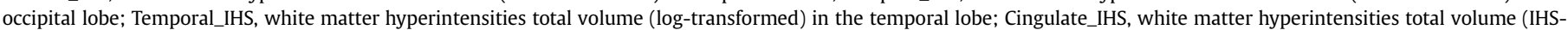
transformed) in the cingulate lobe; Frontal_IHS, white matter hyperintensities total volume (IHS-transformed) in the frontal lobe.

order correlation coefficient between WMH total volume in lobar regions and age (above 43 years old) were frontal $0.46(p<0.0001)$, cingulate $0.39(p<0.0001)$, occipital $0.44(p<0.0001)$, temporal $0.39(p<0.0001)$, and parietal $0.49(p<0.0001)$. Supplementary Table 4 reports the associations between the 5 regional $\mathrm{WMH}$ volumes and the 4 RAs in 4 separate linear regression models using all regional WMH volumes as independent variables and each RA as the dependent variable. Age and education were entered in the model as covariates and accounted for most of the significance in the predictions that were obtained (Supplementary Table 5).

\subsection{PCA between regional WMH and RAs}

We performed PCA to reduce the dimensionality of the regional WMH data. Loadings for each WMH region were extracted for each PC. Loadings and point estimates of PC1-4 can be seen in Supplementary Table 6 . The first PC accounts for $64.58 \%$ of the data variance and the second for the next 16.13\%. Loadings were normalized for all PCs to have an Euclidean norm $=1$. The first PC showed negative loadings in all WMH regions. The second PC showed negative loadings in all WMH regions except in the occipital lobe. These PCs loadings are presented in Table 6 of the supplementary material.

We applied a model of PCR using the obtained PCs to predict each RA separately. For perceptual speed, the model that best predicted cognitive performance consists of 3 PCs. The best model to predict episodic memory across subjects used only the first PC. For fluid reasoning RA, the model best predicting the data variance used the first 2 PCs. For vocabulary, we did not find a significance in any of the models using up to the 5 PCs.

\subsection{Mediation models}

We tested whether WMH mediated between age and cognition. We did not find a mediation effect using WMH total volume. We assessed mediation of each of the 5 regional WMH total volumes and found a significant mediating effect of parietal regional $\mathrm{WMH}$ in the relationship between age and perceptual speed (Supplementary Table 7). No mediation was seen for the other RAs. We then tested multiple mediation models, simultaneously including all 5 regional WMH volumes and controlling for education. In this model, parietal WMH volume mediated approximately 

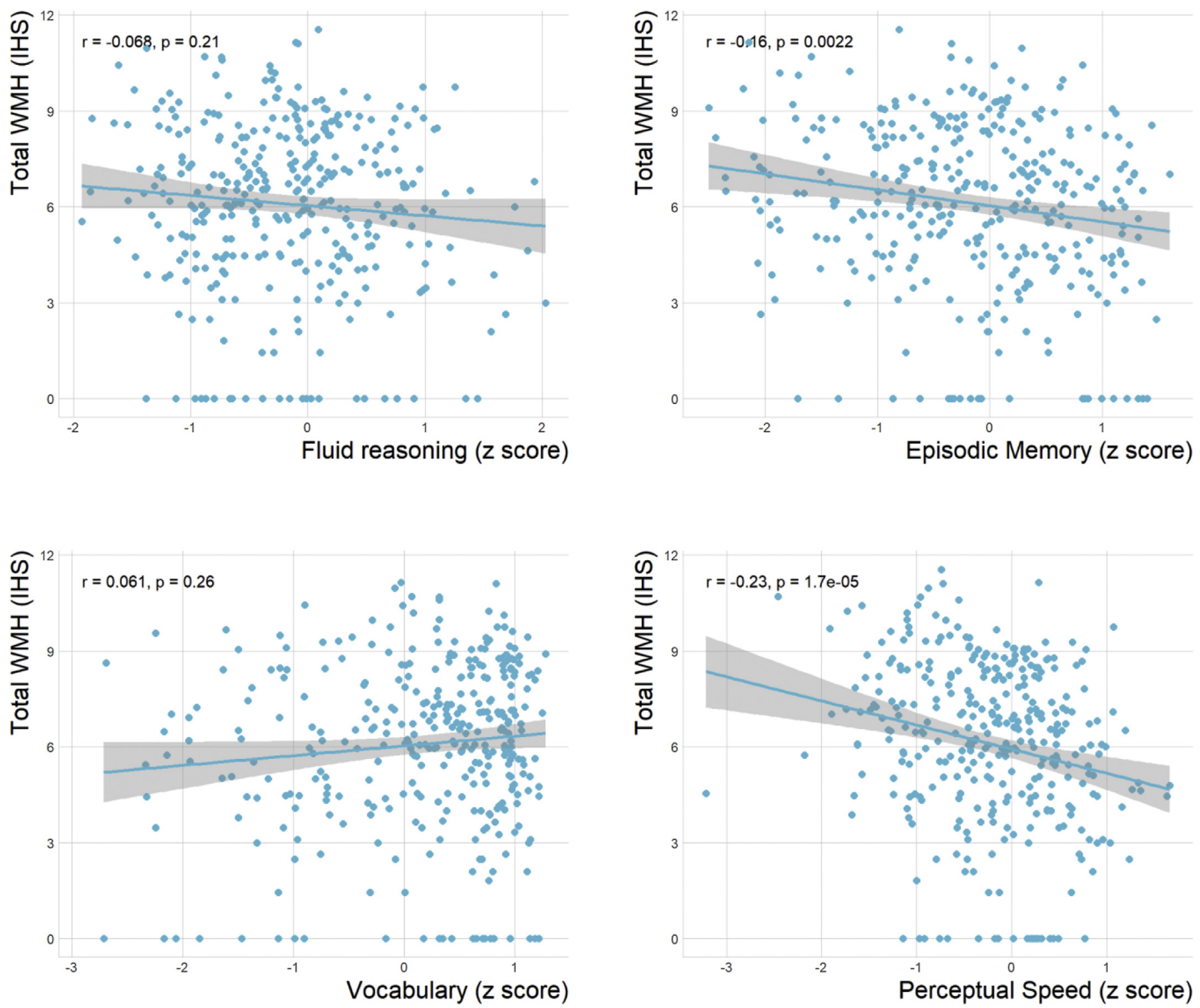

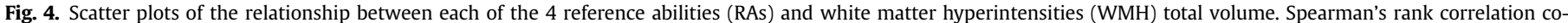

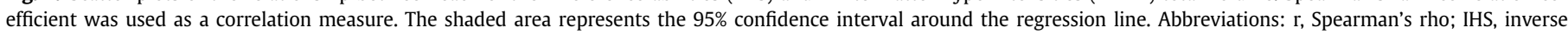
hyperbolic sine.

$25 \%$ of the relation between age and perceptual speed, whereas occipital WMH had suppression effect. The results of this mediation model can be seen in Table 2. Again, there were no multiple mediating effects for the other RAs (Supplementary Tables 8 and 9). We also tested the mediation effect of each pattern of PCR and found that only the pattern for perceptual speed had a significant mediating effect in the relationship between age and perceptual speed, as seen in Fig. 6 (and in Supplementary Table 10).

\section{Discussion}

In this study, we report 3 important findings. First, the estimation of an inflection point where the relationship between age and WMH changes. The second is the relationship between total and regional WMH, as well as, a simultaneous combination of WMH 5 lobar regions, with cognition. The strongest model was for the prediction of perceptual speed; we found a significant prediction of fluid reasoning and memory but not vocabulary. These relationships were not seen after controlling for age. Thirdly, we found that the parietal regional burden of $\mathrm{WMH}$ mediated the relationship between age and perceptual speed RA.

Our study adds information to the growing body of the literature on WMH by describing the prevalence of these common neuroimaging findings across the human life span (Huang et al., 2018). In our larger sample of individuals, with ages across the entire adult life span, we found that around 43 years of age, there appears to be a modification in the relationship between age and the WMH total volume. The finding of an inflection point in a relatively young age converges with the growing evidence of the need to assess components of the aging process starting much earlier than the typical age of onset of neurodegenerative diseases (Debette et al., 2011). This finding also underscores the importance of sampling a wide age range when studying the aging brain. The study of age-related changes in the brain using only older participants increases the chance of missing important nonlinear trends (Raz et al., 2005). There is extensive literature reporting a higher rate of decline with 

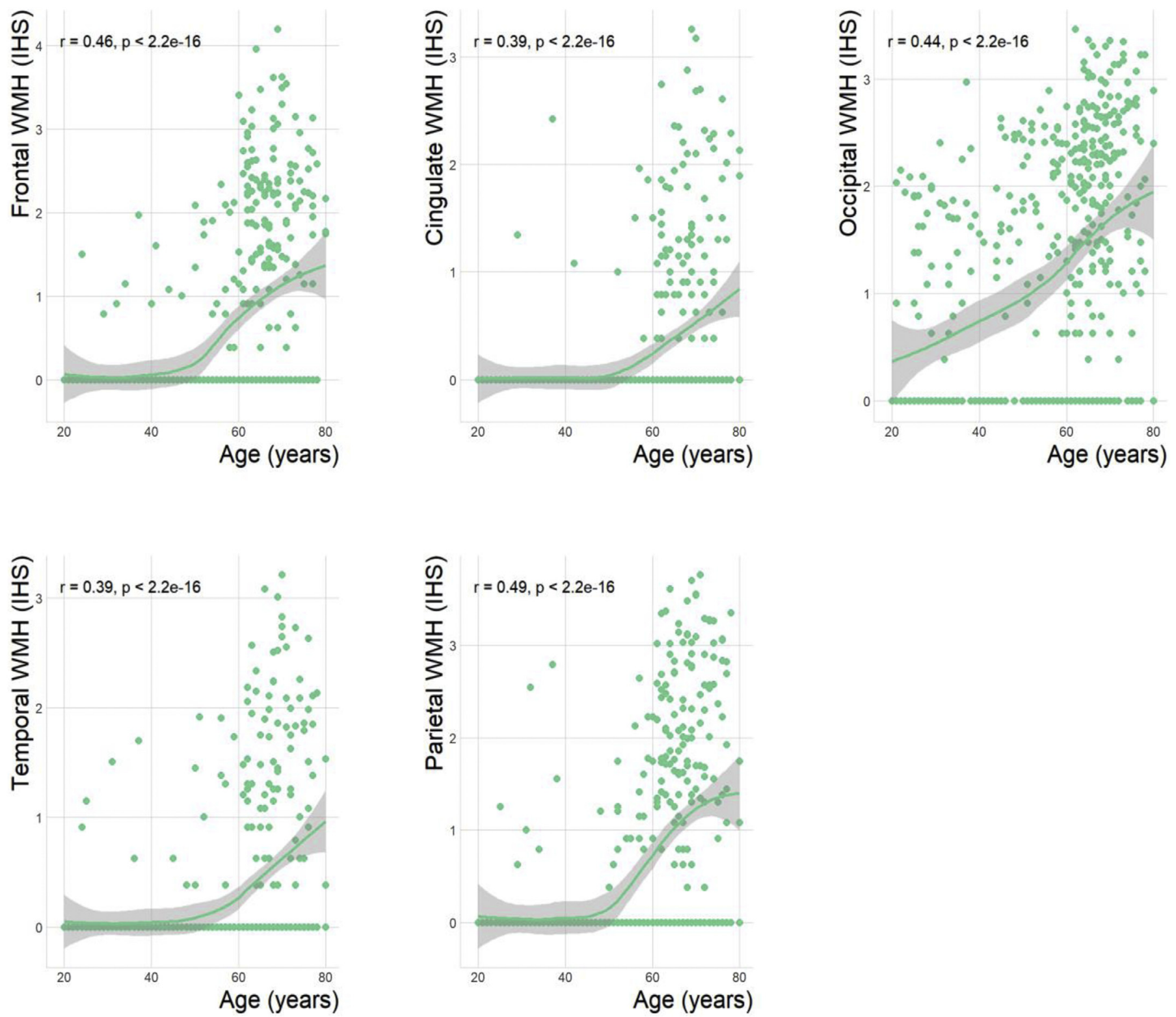

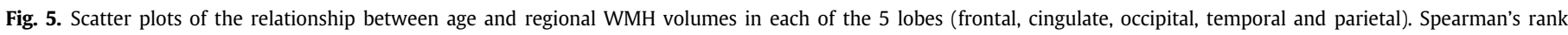

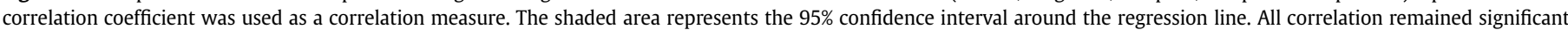
after multiple comparison correction. Abbreviations: IHS, inverse hyperbolic sine; WMH, white matter hyperintensities; $r=$ Spearman's rho.

Table 2

Parameter estimates for multiple mediating effects of WMH regional volumes in the relation between age and perceptual speed

\begin{tabular}{|c|c|c|c|c|c|c|c|}
\hline Effect & Region & Estimate & S.E. & $\mathrm{z}$ & $p$ Value & $95 \% \mathrm{CI}$ & \\
\hline Total & - & -0.02889 & 0.0042 & -6.82 & $<0.0001^{a}$ & -0.0372 & -0.0208 \\
\hline Direct & - & -0.02448 & 0.0044 & -5.54 & $<0.0001^{a}$ & -0.0334 & -0.0159 \\
\hline Indirect & Frontal & -0.00153 & 0.0021 & -0.74 & 0.46 & -0.0056 & 0.0026 \\
\hline \multirow[t]{4}{*}{$\left(a^{*} b\right)$} & Cingulate & -0.00135 & 0.0016 & -0.82 & 0.41 & -0.0046 & 0.0019 \\
\hline & Occipital & 0.00515 & 0.0018 & 2.92 & $0.0035^{a}$ & 0.0022 & 0.0090 \\
\hline & Temporal & 0.00028 & 0.0019 & 0.15 & 0.88 & -0.0034 & 0.0043 \\
\hline & Parietal & -0.00696 & 0.0027 & -2.60 & $0.0092^{a}$ & -0.0124 & -0.0020 \\
\hline
\end{tabular}

The model represents the adjusted total, indirect and direct effects of WMH regional volumes. The $95 \%$ confidence intervals of total, direct and indirect effect were estimated from 10,000 bootstrapped samples. The analysis was run in participants aged more than 43 years.

Bold represents a significance value of $p<0.01$.

Key: WMH, white matter hyperintensities; SE, standard error; 95\% CI, 95\% confidence interval.

${ }^{a}$ Bonferroni corrected $p$-value $<0.05$. aging in some neuroimaging measures including cortical thickness (Raz et al., 2005), (Draganski et al., 2013), brain volume (Raz et al., 2005), and WM tract integrity (Booth et al., 2013). Our crosssectional observation adds information regarding $\mathrm{WMH}$ to these previous reports. Other authors had proposed the age range of midfifties as a likely decade where the point of inflection for some neuroimaging indices, but without formal testing of this hypothesis (Huang et al., 2018; Raz et al., 2005). We estimated an inflection point in where there is a change in the volume of the $\mathrm{WMH}$, observing virtually no extensive WMH burden in young adulthood. This finding may play a vital role, as WMH may be an important biomarker of pathologic changes in aging and disease (Salat et al., 2009).

Cognitive measures are key to unpacking subtle variations between normal cognitive aging and subclinical neurodegenerative processes. Although neuropsychological measures used in most studies are well-validated and quick to administer, typically they 

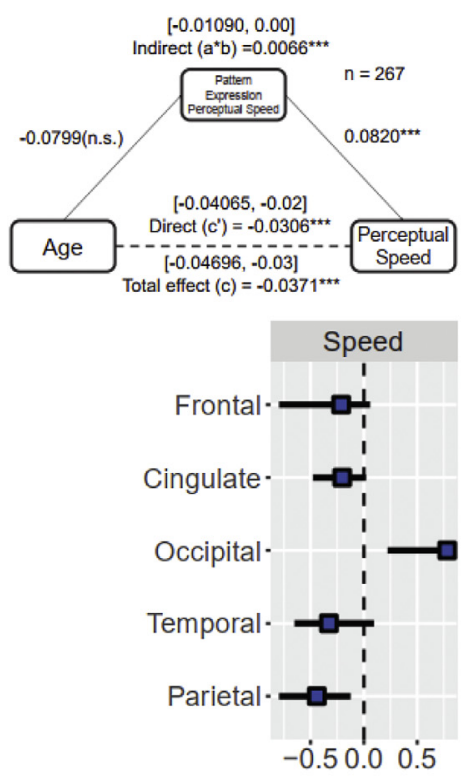
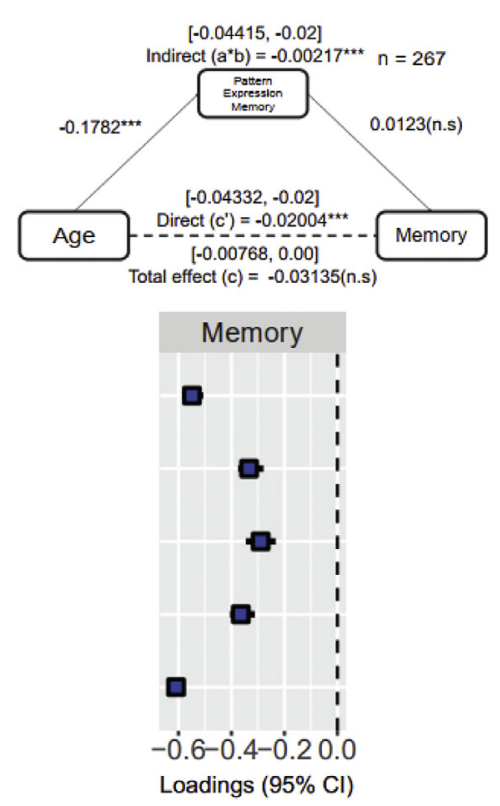
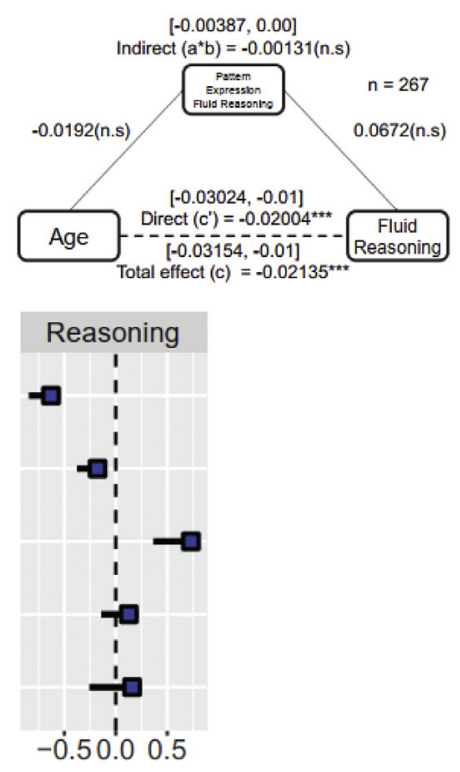

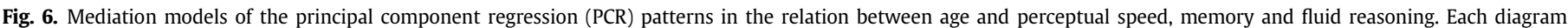

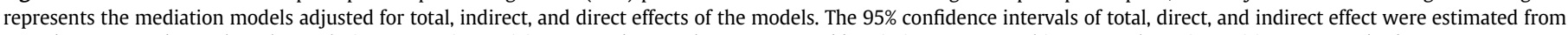

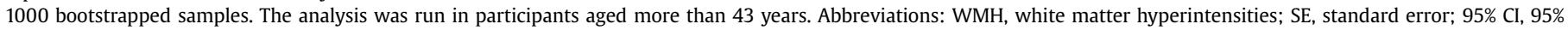
confidence interval. ${ }^{* * *} p<0.01$.

lack the specificity to dissect age-related cognitive processes (Salthouse et al., 2015). Therefore, individual neuropsychological tests have limited use for investigating the direct effect of age on a cognitive phenotype. Numerous studies have assessed the effect of WMH using different sets of neuropsychological batteries, regardless of the association of the cognitive domains with age. A more selective assessment of cognitive domains may be advantageous, in the context of the study of aging and age-related neuroimaging findings. Indeed, the RA construct presents as a very robust method to address cognitive variation across the life span specifically in older adults because these 4 reference abilities have been demonstrated to capture most of the age-related variance in cognition. One of the main strengths of our approach is, thus, a comprehensive assessment of brain function using neuropsychological constructs which are proposed to be better suited to capture cognitive aging (Salthouse, 2009).

Although we noted robust correlations between WMH total burden and cognition, these relationships were no longer significant after controlling for age. Because age and WMH burden is so strongly correlated, it is difficult to determine the unique effect of WMH on cognition in this context. Thus, the mediation models were of great importance because they assess whether the effect of age on cognition may be mediated by WMH. We evaluated the impact of the regional distribution of WMH on specific cognitive domains by using a mediation model to study the effect of total and regional WMH on the 4 RAs individually. Interestingly, we found that the WMH total volume alone did not mediate the relationship between age and each of the RAs. We in fact found that parietal WMH regional volume mediated the association between age and worse performance in perceptual speed. When all regions were entered in the mediation model, parietal WMH still contributed significantly, but a suppression effect was noted for occipital WMH. Because occipital WMH volume did not mediate on its own, we attribute this suppression effect to some artifact of the multiple variables included in the regression. One possibility is that different age trajectory of the occipital lobe WMH burden contributed to this result. This should be followed up in an independent data set.
Previous literature had observed a mediating effect between age, WMH total volume, brain atrophy, and global measures of cognition. The present study further explores the cognitive implications of $\mathrm{WMH}$ in specific cognitive domains.

From a methodological perspective, canonical correlation, PCR, and its variants (such as partial least squares correlation) can be used in neuroimaging studies to explore structural MRI findings. Our approach was a direct application of this method using a PCR to evaluate the relationship of regional WMH to each of the 4 RAs. The method was tuned using feature selection to optimize the prediction of RA scores using the 5 regional WMH volumes. This technique improves on simple multiple linear regression because it accounts for correlation patterns between the independent variables. This methodology provides a unique contribution to the literature because it is one of the first attempts to study the relationship between the different regions where WMH appear and their specific relationship with cognition. In the present study, we found selective associations between WMH regional volumes and specific RAs. Perceptual speed provided the best model and it was derived using a linear combination of PCs 1-3, implying a negative relationship between speed performance and the 5 regional $\mathrm{WMH}$ volumes. In addition, the derived PCR pattern for perceptual speed has a mediating effect on the relationship between age and perceptual speed RA. Even using the PCA approach, we saw no association between vocabulary and the PCs scores.

Although the present study has several strengths, including a large population-based sample, use of effective cognitive measures to study aging, and powerful statistical modeling procedures, to deal with the typical limitations of these measurements, we should acknowledge some limitations. Our ability to infer causality is limited by the cross-sectional design. Historically, the study of the aging brain is frequently based on results drawn from cross-sectional studies (Raz et al., 2005). The conclusions from most studies rely on correlations between age and the specific variable of interest, which deems the possibility to directly report the rate of change associated with age in the same individual differences. We are therefore very cautious in assuming any causality with these results. 
In summary, our results stress the importance of studying the entire adult life span to explore the effects of aging on the brain. As WMH are presumed to be MRI correlates of small vessel cerebrovascular disease, our findings support the claims of emerging importance in the reduction of cardiovascular risk cardiovascular (CV) factors in adult life. In fact, clinical researchers focused on neurodegenerative disease, recently started assessing midlife interventions to promote healthy aging (Ecay-Torres et al., 2018). Moreover, most studies of WMH in mid adulthood originate from the fields of $\mathrm{CV}$ clinical research and, as an expected consequence, cognitive measurement is far from the ideal to study cognitive aging (Pase et al., 2016). On the other hand, studies in older adults have more extensive cognitive assessments but do not explore the modification in WMH patterns across the life span. Our work includes adults across a wide age range while assessing cognition with very robust constructs to study aging. To tackle nonlinearity problems, we used 2 different techniques to explore the relationship between age, WMH, and RAs. PCA as an exploratory approach and PCR as a predictive model to study the possible cognitive differences dependent on the regional burden of WMH. Finally, mediation models to further assess the effect of WMH in the relationship between age and each of the 4 RAs individually are a powerful approach to dealing with the multiple and intricate relationship between cognitive performance and the effect of aging.

In all, because WMH seems to represent a proxy of vascular disease in the brain, their study in the context of aging and outside the scope of dementia is important. By tuning our methods to determine critical regions in the brain, where and when these lesions might start affecting cognition, we might contribute to further dissecting the concept of age-related cognitive decline. In fact, if these once considered normal and ubiquitous lesions in older adults could be instead preventable by the same interventions that reduce $\mathrm{CV}$ risk factors, this could help define what healthy cognitive aging really is (Hörder et al., 2018).

\section{Disclosure}

The authors report no competing interests.

\section{Acknowledgements}

The authors thank David Predovan, PhD, for comments that greatly improved the article.

This work was supported by the National Institute on Aging [Grant numbers R01 AG026158 and RF1AG038465].

\section{Appendix A. Supplementary data}

Supplementary data to this article can be found online at https:// doi.org/10.1016/j.neurobiolaging.2019.08.024.

\section{References}

Belotti, F., Deb, P., Manning, W., Norton, E., 2015. twopm: two-part models. Stata J. $15,3-20$.

Booth, T., Bastin, M.E., Penke, L., Maniega, S.M., Murray, C., Royle, N.A., Gow, A.J., Corley, J., Henderson, R.D., Hernandez Mdel, C., Starr, J.M., Wardlaw, J.M., Deary, I.J., 2013. Brain white matter tract integrity and cognitive abilities in community-dwelling older people: the Lothian Birth Cohort, 1936. Neuropsychology 27, 595-607.

Brickman, A.M., Muraskin, J., Zimmerman, M.E., 2009. Structural neuroimaging in Alzheimer's disease: do white matter hyperintensities matter? Dialogues Clin. Neurosci. 11, 181-190.

Brickman, A.M., Provenzano, F.A., Muraskin, J., Manly, J.J., Blum, S., Apa, Z., Stern, Y., Brown, T.R., Luchsinger, J.A., Mayeux, R., 2012. Regional white matter hyperintensity volume, not hippocampal atrophy, predicts incident Alzheimer disease in the community. Arch. Neurol. 69, 1621-1627.
Brickman, A.M., Zahodne, L.B., Guzman, V.A., Narkhede, A., Meier, I.B., Griffith, E.Y., Provenzano, F.A., Schupf, N., Manly, J.J., Stern, Y., Luchsinger, J.A., Mayeux, R., 2015. Reconsidering harbingers of dementia: progression of parietal lobe white matter hyperintensities predicts Alzheimer's disease incidence. Neurobiol. Aging $36,27-32$.

Burbidge, J.B., Magee, L., Robb, A.L., 1988. Alternative transformations to handle extreme values of the dependent variable. J. Am. Stat. Assoc. 83, 123-127.

Buschke, H., Fuld, P.A., 1974. Evaluating storage,retention, and retrieval in disordered memory and learning. Neurology 24, 1019-1025.

de Groot, J., de Leeuw, F., Oudkerk, M., Hofman, A., Jolles, J., Breteler, M., 2001. Cerebral white matter lesions and subjective cognitive dysfunction. Rotterdam Scan Study. Neurology 56, 1539-1545.

De Groot, J.C., De Leeuw, F.E., Oudkerk, M., Van Gijn, J., Hofman, A., Jolles, J., Breteler, M.M.B., 2002. Periventricular cerebral white matter lesions predict rate of cognitive decline. Ann. Neurol. 52, 335-341.

De Leeuw, F.E., De Groot, J.C., Achten, E., Oudkerk, M., Ramos, L.M., Heijboer, R. Hofman, A., Jolles, J., van Gijn, J., Breteler, M.M., 2001. Prevalence of cerebral white matter lesions in elderly people: a population based magnetic resonance imaging study. The Rotterdam Scan Study. J. Neurol. 70, 9-14.

Debette, S., Seshadri, S., Beiser, A., Au, R., Himali, J.J., Palumbo, C., Wolf, P.A. DeCarli, C., 2011. Midlife vascular risk factor exposure accelerates structural brain aging. Neurology 77, 461-468.

DeCarli, C., Massaro, J., Harvey, D., Hald, J., Tullberg, M., Au, R., Beiser, A., D'Agostino, R., Wolf, P.A., 2005. Measures of brain morphology and infarction in the framingham heart study: establishing what is normal. Neurobiol. Aging 26, $491-510$.

Desikan, R.S., Ségonne, F., Fischl, B., Quinn, B.T., Dickerson, B.C., Blacker, D., Buckner, R.L., Dale, A.M., Maguire, R.P., Hyman, B.T., Albert, M.S., Killiany, R.J., 2006. An automated labeling system for subdividing the human cerebral cortex on MRI scans into gyral based regions of interest. Neuroimage 31, 968-980.

Draganski, B., Lutti, A., Kherif, F., 2013. Impact of brain aging and neurodegeneration on cognition. Curr. Opin. Neurol. 26, 640-645.

Ecay-Torres, M., Estanga, A., Tainta, M., Izagirre, A., Garcia-Sebastian, M., Villanua, J. Clerigue, M., Iriondo, A., Urreta, I., Arrospide, A., Díaz-Mardomingo, C. Kivipelto, M., Martinez-Lage, P., 2018. Increased CAIDE dementia risk, cognition, CSF biomarkers, and vascular burden in healthy adults. Neurology 91, e217-e226.

Fischl, B., Salat, D.H., Busa, E., Albert, M., Dieterich, M., Haselgrove, C., Van Der Kouwe, A., Killiany, R., Kennedy, D., Klaveness, S., Montillo, A., Makris, N. Rosen, B., Dale, A.M., 2002. Whole brain segmentation: automated labeling of neuroanatomical structures in the human brain. Neuron 33, 341-355.

Frisoni, G.B., Galluzzi, S., Pantoni, L., Filippi, M., 2007. The effect of white matter lesions on cognition in the elderly-small but detectable. Nat. Clin. Pract. Neurol. 3, 620-627.

Golden, C.J., 1978. Stroop Color and Word Test: A Manual for Clinical and Experimental Uses. Stoelting, Chicago, pp. 1-46.

Grober, E., Sliwinski, M., 1991. Development and validation of a model for estimating premorbid verbal intelligence in the elderly. J. Clin. Exp. Neuropsychol. 13, 933-949.

Habeck, C., Foster, N.L., Perneczky, R., Kurz, A., Alexopoulos, P., Koeppe, R.A. Drzezga, A., Stern, Y., 2008. Multivariate and univariate neuroimaging biomarkers of Alzheimer's disease. Neuroimage 40, 1503-1515.

Habeck, C., Gazes, Y., Razlighi, Q., Steffener, J., Brickman, A., Barulli, D., Salthouse, T. Stern, Y., 2016. The Reference Ability Neural Network Study: life-time stability of reference-ability neural networks derived from task maps of young adults. Neuroimage 125, 693-704.

Hörder, H., Johansson, L., Guo, X., Grimby, G., Kern, S., Östling, S., Skoog, I., 2018 Midlife cardiovascular fitness and dementia. Neurology 90, e1298-e1305.

Huang, C.C., Yang, A.C., Chou, K.H., Liu, M.E., Fang, S.C., Chen, C.C., Tsai, S.J., Lin, C.P., 2018. Nonlinear pattern of the emergence of white matter hyperintensity in healthy Han Chinese: an adult lifespan study. Neurobiol. Aging 67, 99-107.

Iadecola, C., 2013. The pathobiology of vascular dementia. Neuron 80, 844-866.

Lee, S., Habeck, C., Razlighi, Q., Salthouse, T., Stern, Y., 2016. Selective association between cortical thickness and reference abilities in normal aging. Neuroimage 142, 293-300.

Lindemer, E.R., Greve, D.N., Fischl, B., Augustinack, J.C., Salat, D.H., 2017a. Differential regional distribution of Juxtacortical white matter signal abnormalities in aging and Alzheimer's disease. J. Alzheimers Dis. 57, 293-303.

Lindemer, E.R., Greve, D.N., Fischl, B.R.,Augustinack, J.C. Salat, D.H. 2017b. Regional staging of white matter signal abnormalities in aging and Alzheimer's disease. Neuroimage Clin. 14, 156-165.

Longstreth, W.T., Manolio, T.A., Arnold, A., Burke, G.L., Bryan, N., Jungreis, C.A. Enright, P.L., O'Leary, D., Fried, L., 1996. Clinical correlates of white matter findings on cranial magnetic resonance imaging of 3301 elderly people: the cardiovascular health study. Stroke 27, 1274-1282.

Mattis, S., 1988. Dementia Rating Scale. Psychological Assessment Resources Inc, Odessa, F.L.

McGee, V.E., Carleton, W.T., 1970. Piecewise regression. J. Am. Stat. Assoc. 65 1109-1124.

Pase, M.P., Himali, J.J., Mitchell, G.F., Beiser, A., Maillard, P., Tsao, C., Larson, M.G., Decarli, C., Vasan, R.S., Seshadri, S., 2016. Association of aortic stiffness with cognition and brain aging in young and middle-aged adults: the framingham third generation cohort study. Hypertension 67, 513-519.

Piepho, H.P., Ogutu, J.O., 2003. Inference for the break point in segmented regression with application to longitudinal data. Biometrical J. 45, 591-601. 
Prins, N.D., Van Dijk, E.J., Den Heijer, T., Vermeer, S.E., Jolles, J., Koudstaal, P.J., Hofman, A., Breteler, M.M.B., 2005. Cerebral small-vessel disease and decline in information processing speed, executive function and memory. Brain 128, 2034-2041.

Raz, N., Lindenberger, U., Rodrigue, K.M., Kennedy, K.M., Head, D., Williamson, A., Dahle, C., Gerstorf, D., Acker, J.D., 2005. Regional brain changes in aging healthy adults: general trends, individual differences and modifiers. Cereb. Cortex 15, 1676-1689.

Reitan, R., 1958. Validity of the Trail Making Test as an indicator of organic brain damage. Percept. Mot. Skills 8, 271-276.

Salat, D.H., Lee, S.Y., van der Kouwe, A.J., Greve, D.N., Fischl, B., Rosas, H.D., 2009. Age-associated alterations in cortical gray and white matter signal intensity and gray to white matter contrast. Neuroimage 48, 21-28.

Salthouse, T.A., 2005. Relations between cognitive abilities and measures of executive functioning. Neuropsychology 19, 532-545.

Salthouse, T.A., 2009. Decomposing age correlations on neuropsychological and cognitive variables. J. Int. Neuropsychological Soc. 15, 650-661.

Salthouse, T.A., Habeck, C., Razlighi, Q., Barulli, D., Gazes, Y., Stern, Y., 2015. Breadth and age-dependency of relations between cortical thickness and cognition. Neurobiol. Aging 36, 3020-3028.

Schmidt, P., Gaser, C., Arsic, M., Buck, D., Förschler, A., Berthele, A., Hoshi, M., Ilg, R., Schmid, V.J., Zimmer, C., Hemmer, B., Mühlau, M., 2011. An automated tool for detection of FLAIR-hyperintense white-matter lesions in multiple sclerosis. Neuroimage 59, 3774-3783.
Smith, E.E., Jeng, J., Fischl, B., Blacker, D., 2011. Correlations between MRI white matter lesion location and executive function and episodic memory. Neurology 76, 1492-1499.

Stern, Y., 2012. Cognitive reserve in ageing and Alzheimer's disease. Lancet Neurol. 11, 1006-1012.

Stern, Y., Habeck, C., Steffener, J., Barulli, D., Gazes, Y., Razlighi, Q., Shaked, D. Salthouse, T., 2014. The reference ability neural network study: motivation, design, and initial feasibility analyses. Neuroimage 103, 139-151.

van Dijk, E.J., Prins, N.D., Vermeer, S.E., Koudstaal, P.J., Breteler, M.M.B., 2002. Frequency of white matter lesions and silent lacunar infarcts. J. Neural Transm. Suppl. 25-39.

Wechsler, D., 1997. Wechsler Adult Intelligence Scale (WAIS-3R). Psychologica Corporation, San Antonio, TX.

Venegas, J., Clark, E., 2011. Wechsler Test of Adult Reading. In: Kreutzer, J.S, DeLuca, J., Caplan, B. (Eds.), Encyclopedia of Clinical Neuropsychology. Springer, New York, NY.

Wiegman, A.F. Meier, I.B., Provenzano, F.A. Schupf, N., Manly, J.J., Stern, Y., Luchsinger, J.A., Brickman, A.M., 2013. Regional white matter hyperintensity volume and cognition predict death in a multiethnic community cohort of older adults. J. Am. Geriatr. Soc. 61, 2246-2248.

Yoshita, M., Fletcher, E., Harvey, D., Ortega, M., Martinez, O., Mungas, D.M., Reed, B.R., DeCarli, C.S., 2006. Extent and distribution of white matter hyperintensities in normal aging, MCI, and AD. Neurology 67, 2192-2198. 